\title{
Ring finger 20/ring finger 40/WW domain-containing adaptor with coiled-coil complex interacts with p53 to regulate gene transcription in DNA damage response
}

\author{
DANNI MENG ${ }^{*}$, KUN GUO*, DIE ZHANG ${ }^{*}$, CHENG ZHAO, CHUANWEN SUN and FENG ZHANG \\ College of Life Sciences, Shanghai Normal University, Shanghai 200234, P.R. China
}

Received January 19, 2020; Accepted December 17, 2020

DOI: $10.3892 / \mathrm{ol} .2021 .12697$

\begin{abstract}
. p53 is one of the most important tumor suppressor genes, and its primary function is to act as a transcriptional activator to control cell cycle arrest, DNA repair and cellular metabolism by recognizing and binding to specific DNA sequences. Defects in the ring finger (RNF)20/RNF40/WW domain-containing adaptor with coiled-coil (WAC) complex, one of the histone H2B ubiquitination E3 ligases, have been reported to be a key factor in oncogenesis, cancer cell migration and invasion. Histone H2B mono-ubiquitination has been demonstrated to be essential for maintaining the functionality of the p53 tumor suppressor protein. The aim of the present study was to identify any sites in the p53 DNA-binding domain (DBD) specific to the RNF20/RNF40/WAC complex that may be involved in the gene regulation in DNA damage response. The results demonstrated that p53 and the RNF20/RNF40/WAC complex interacted with each other, and the coiled-coil regions in RNF20, RNF40 and WAC were identified to directly interact with p53. The R282 site in the p53 DBD, one of the frequent missense mutations associated with p53 mutation-dependent cancer, was demonstrated to be the key binding site for the RNF20/RNF40/WAC complex. Furthermore, knockout of RNF20/RNF40 suppressed the expression levels of p53 and its target genes in HCT116 cells compared with those in wild-type HCT116 cells. Consistent with these results, the $\mathrm{R} 282 \mathrm{~W}$ mutation in p53 inhibited the expression levels of p53 and its downstream genes by inactivating the interaction between p53 and RNF20/RNF40 compared with those in wild-type HCT116 cells. In conclusion,
\end{abstract}

Correspondence to: Dr Chuanwen Sun or Professor Feng Zhang, College of Life Sciences, Shanghai Normal University, 100 Guilin Road, Shanghai 200234, P.R. China

E-mail: sunwill12@163.com

E-mail: fengz@shnu.edu.cn

${ }^{*}$ Contributed equally

Key words: ring finger 20/ring finger 40/WW domain-containing adaptor with coiled-coil complex, coiled-coil domain, p53, p53 missense mutation R282W, gene transcription the results of the present study revealed the molecular mechanism of the interaction between the RNF20/RNF40/WAC complex and $\mathrm{p} 53$, and demonstrated that these proteins regulated gene transcription in the DNA damage response.

\section{Introduction}

p53 is a tumor suppressor protein (1). Mutations within p53 have been demonstrated to lead to its inactivation, which is associated with $\sim 50 \%$ of all types of human cancer (2). The p53 protein contains an amino N-terminal transactivation domain, a proline-rich domain, a central DNA-binding domain (DBD), a tetramerization domain and a carboxy-terminal regulatory domain (CRD) (3). The majority of the cancer-associated mutations are missense mutations located within the DBD (4). R175H, Y220C, G245S, R248Q/W, R249S, R273C/H and $\mathrm{R} 282 \mathrm{~W}$ are high-frequency mutations that alter protein conformation and attenuate sequence-specific binding to proteins (5). This results in the inhibition of wild-type (WT) p53 function, and the mutated p53 interacts with inappropriate proteins and stimulates oncogenic genes (6). p53 is also an important transcription factor involved in the regulation of DNA repair, cell cycle arrest and apoptosis (7). Under normal conditions, p53 is present in a steady state, whereas in cells undergoing DNA damage or abnormal oncogenic events, p53 is activated through post-translational modifications, such as phosphorylation, ubiquitination and acetylation (8). Therefore, the levels of p53 are increased, resulting in the transactivation of the downstream target genes, which are involved in cell cycle arrest, DNA repair, autophagy and cellular metabolism (4). Furthermore, p53 activates genes such as AMP-activated protein kinase $\beta$, tuberin and PTEN to suppress the mTOR (nutrient sensor) signaling pathway, which participates in aerobic glycolysis and oxidative phosphorylation (9). In addition, cytosolic p53 exerts transcription-independent functions, including triggering apoptosis by interacting with the apoptotic effector proteins BAX and BAK, and repressing autophagy by inhibiting the positive autophagy regulator AMP-dependent kinase (10-12).

In eukaryotic cells, genomic DNA is packaged into chromatin and encapsulates histone octamers to form nucleosomes (13). The $\mathrm{N}$ - and C-termini of histones can be covalently modified by methylation, acetylation and ubiquitination (14). Histone ubiquitination is an important epigenetic 
modification widely involved in the regulation of chromatin structure, gene transcription, the cell cycle and other physiological processes (15). Ubiquitination is one of the covalent post-translational modifications, during which the $8-\mathrm{kD}$ ubiquitin molecule (mono-ubiquitination) or a poly-ubiquitin chain (poly-ubiquitination) conjugate to a protein substrate (16). The ubiquitin E3 ligase functions in the last step of the ubiquitination cascade (17). The ring finger (RNF) E3 enzyme, RNF20/RNF40 dimer, catalyzes the histone H2B mono-ubiquitination (H2Bub1) at lysine 120 in the C-terminus (18). $\mathrm{H} 2 \mathrm{Bub} 1$ is essential for maintaining functionality of the p53 tumor suppressor protein (19). The loss of RNF20 and RNF40 attenuates the p53-dependent cell response to cellular stress or toxicity; for example, RNF20-knockdown by RNA interference in HeLa cells leads to decreased apoptosis and impaired cell cycle arrest compared with those in control-transfected HeLa cells (20). In addition, WAC has been reported to act as a functional partner of the RNF20/RNF40 dimer, which mediates the interaction of the RNF20/RNF40 dimer with RNA polymerase II $(21,22)$. Furthermore, p53 has been reported to interact with the RNF20/RNF40/WAC complex directly and to recruit the complex for $\mathrm{H} 2 \mathrm{Bub} 1$ to target the p53 gene loci $(21,23)$.

An increasing number of studies have demonstrated that the RNF20/RNF40/WAC complex is associated with genomic stability and tumorigenesis $(8,24-26)$. Therefore, it is important to investigate the underlying molecular mechanism by which the RNF20/RNF40/WAC complex and p53 synergistically regulate target gene transcription. A previous study by $\mathrm{Wu}$ et al (27) reported that the CRD of p53 is a key binding region with the RNF20/RNF40 dimer; however, only partial deletion mutants of the p53 DBD were constructed in their study. There is currently no evidence of specific sites interacting with the RNF20/RNF40 dimer close to the C-terminal region of the p53 DBD. In the present study, we hypothesized that, in addition to the CRD region interacting with RNF20, the amino acid (AA)201-AA300 region of the p53 DBD may also interact with RNF20/RNF40, and that one of the cancer-related hotspot sites of p53 may be a potential site for the interaction with the RNF20/RNF40 dimer. The aim of the present study was to detect the specific binding site of p53 with the RNF20/RNF40 dimer and to investigate the gene regulation function of these sites.

\section{Materials and methods}

Cell culture. The 293T, U2OS and HCT116 cell lines were obtained from the Shanghai Institute of Biochemistry and Cell Biology, and cultured in Dulbecco's modified Eagle's medium (DMEM; Sigma-Aldrich; Merck KGaA) with 10\% fetal bovine serum (Gibco; Thermo Fisher Scientific, Inc.), and $1 \%$ streptomycin and penicillin. The cells were cultured at $37^{\circ} \mathrm{C}$ in a humidified incubator with $5 \% \mathrm{CO}_{2}$. All cell lines were confirmed to be free from mycoplasma contamination.

Co-immunoprecipitation (Co-IP) and western blot assays. After washing with $1 \mathrm{X}$ PBS, the cells were lysed with the NETN-100 buffer [150 mM NaCl, 0.2 mM EDTA, 1\% NP-40 and $50 \mathrm{mM}$ Tris- $\mathrm{HCl}(\mathrm{pH} 7.5)]$ for 10 min on ice, supplemented with $1 \mathrm{X}$ protease inhibitor cocktail (Merck KGaA) and $1 \mathrm{mM}$ phenylmethylsulfonyl fluoride, and centrifuged at 12,000 x g at $4^{\circ} \mathrm{C}$ for $10 \mathrm{~min}$. For Co-IP, the cell lysates were incubated with $50 \mu \mathrm{l}$ protein $\mathrm{A} / \mathrm{G}$ agarose (Sangon Biotech Co., Ltd.) and the following antibodies (dilution, 1:1,000 for western blotting and 1:500 for Co-IP): Anti-HA (cat. no. D199961-0100; Sangon Biotech Co., Ltd.), anti-p53 (cat. no. ab26; Abcam), anti-FLAG (cat. no. 14793; Cell Signaling Technology, Inc.), anti-WAC (cat. no. ABE471; Merck KGaA), anti-rat IgG (cat. no. ab172730; Abcam), anti-RNF20 (cat. no. A300-714A; Bethyl Laboratories, Inc.) and anti-RNF40 (cat. no. Q2680124; Bethyl Laboratories, Inc.) overnight at $4^{\circ} \mathrm{C}$. Following incubation, precipitates were washed three times with PBS and added to the NETN-100 buffer. Whole cell lysates and washed precipitates were incubated at $100^{\circ} \mathrm{C}$ for $8 \mathrm{~min}$ in a dry bath incubator (Tiangen Biotech Co., Ltd) and separated by $10 \%$ SDS-PAGE. The membranes were incubated with the aforementioned antibodies, followed by goat anti-mouse IgG (H+L) HRP-conjugated (cat. no. AP308P; Sigma-Aldrich; Merck KGaA) and goat anti-rabbit IgG H\&L HRP-conjugated (cat. no. ab205718; Abcam) secondary antibodies (dilution, 1:1,000) at room temperature for $1.5 \mathrm{~h}$. The membranes were incubated with SuperSignal West Pico Chemiluminescent Substrate (Thermo Fisher Scientific, Inc.) and visualized using an X-ray film or an automatic digital imaging system. A total of three independent experiments were performed. The semi-quantitative analysis of the western blotting results was performed using ImageJ 1.42q software Java 1.6.0_12(64-bit) (National Institutes of Health) and GraphPad Prism version 6.02 for Windows (GraphPad Software, Inc.).

Mutant construction and plasmid transfection. Human full-length RNF20, RNF40, RNF20 deletion 1 (D1)-D8 and RNF40 D1-D14 were cloned into the HA-tagged vector modified from pCDNA 3.1 (Thermo Fisher Scientific, Inc.). Human full-length, internal deletion ( $\Delta$ aa. 101-200 and $\Delta$ aa. 201-300) and missense mutation sequences of p53 (R248W, R248Q, R249S, R273C, R273H, R282W and R248QR273C) were cloned into the pCDNA3.1-HA vector. Human full-length and mutated WAC deletion (WD)1-7 were cloned into the pS-FLAG-SBP vector (Addgene, Inc.). Deletion mutations within RNF20, RNF40 and WAC, and missense mutations in p53, were generated using the QuikChange site-directed mutagenesis kit (Agilent Technologies, Inc.). Constructed plasmids (5 $\mu \mathrm{g}$ for cells incubated in a $10-\mathrm{cm}$ dish) with the indicated mutations were transfected into U2OS cells (for RNF20/RNF40/WAC deletion mutations generation) and $293 \mathrm{~T}$ cells (for p53 deletion and missense mutations generation) at $70-80 \%$ confluence using ViaFect (Promega Corporation) and Opti-MEM (Thermo Fisher Scientific, Inc.) without FBS for 4-6 $\mathrm{h}$ according to the manufacturers' instructions. Subsequently, the cells were washed with PBS and cultured in DMEM with $10 \%$ FBS and $1 \%$ streptomycin and penicillin for $24 \mathrm{~h}$ prior to co-IP and western blot assays. The single amino acid mutations were constructed according to previous studies by Tzin et al (28) and Kitzman et al (29), whereas the deletion mutations were constructed according to previous studies by Zhang and Yu (21).

Reverse transcription-quantitative PCR (RT-qPCR). Total RNA was extracted from WT, RNF20 $0^{-/}$and RNF40 ${ }^{-/-}$HCT116 cell lines using TRIzol ${ }^{\circledR}$ reagent (Thermo Fisher Scientific, 
Inc.) according to the manufacturer's instructions. RNA concentration was measured using a microplate reader. RT of the total RNA was performed using the PrimeScript ${ }^{\mathrm{TM}} 1 \mathrm{st}$ Strand cDNA Synthesis kit (Takara Bio, Inc.). qPCR was performed with gene-specific primers and the QuantiNova SYBR ${ }^{\circledR}$ Green PCR kit (Qiagen, Inc.) on a CFX96 Real-Time PCR Detection system (Bio-Rad Laboratories, Inc.) The primers used were as follows: GAPDH forward, 5'-ACCCACT CCTCCACCTTTGA-3' and reverse, 5'-CTGTTGCTGTAG CCAAATTCGT-3'; p53 forward, 5'-AGATGGGGTCTC ACAGTGTTGC-3' and reverse, 5'-ATGTTGACCCTTCCA GCTCCAC-3'; p21 forward, 5'-CATGCCAGCTACTTC CTCCT-3' and reverse, 5'-CAGGTCTGAGTGTCCAGGAA-3'; p53-upregulated modulator of apoptosis (PUMA) forward, 5'-GACGACCTCAACGCACAGTA-3' and reverse, 5'-CTA ATTGGGCTCCATCTCG-3'; Achaete-Scute homolog 1 (mash1) forward, 5'-CGACTTCACCAACTGGTTCTG-3' and reverse, 5-ATGCAGGTTGTGCGATCA-3'; and octamerbinding protein 4 (Oct4) forward, 5'-CGCAAGCCCTCA TTTCAC-3' and reverse, 5'-CATCACCTCCACCACCTG-3'. The following thermocycling conditions were used: Initial denaturation at $95^{\circ} \mathrm{C}$ for $2 \mathrm{~min}$, followed by 35-40 cycles at $95^{\circ} \mathrm{C}$ for $5 \mathrm{sec}$ and $60^{\circ} \mathrm{C}$ for $10 \mathrm{sec}$. The $2^{-\Delta \Delta \mathrm{Cq}}$ method was used for quantification (30). A total of three independent experiments were performed.

Cell line construction using CRISPR-Cas9. The primers for the deletion of RNF20 or RNF40 were designed from the CRISPR website (http://crispr.mit.edu/) and confirmed for accuracy using the Cas OFFinder website (http://www. rgenome.net/cas-offinder/). The following primers were used: CRISPR-RNF20-CDS2 forward, 5'-CACCGTATTGAT TGTCAACCGATAC-3' and reverse, 5'-AAACGTATCG GTTGACAATCAATAC-3'; and CRISPR-RNF40-CDS2 forward, 5'-CACCGTCCTCATCGTCAATCGCTAC-3' and reverse, 5'-AAACGTAGCGATTGACGATGAGGAC-3'. The pSpCas9(BB)-2A-Pure V2.0 (PX49) vector (Addgene, Inc.) was used, while Lipofectamine 2000 (Thermo Fisher Scientific, Inc.) was used for plasmid transfection. A total of $2.5 \mu \mathrm{g} /$ well plasmid was transfected into HCT116 cells at $70-80 \%$ confluence in a 6 -well plate for $4-6 \mathrm{~h}$ at $37^{\circ} \mathrm{C}$. Subsequently, the cells were cultured using a concentration gradient and treated with $1 \mu \mathrm{g} / \mathrm{ml}$ puromycin for 3 days to screen the successfully transfected cells. Following 2-week culture, 12 monoclones with expected RNF20 deletion and 12 monoclones with expected RNF40 deletion were selected and expanded for further culture. Finally, the protein knockout effect was detected using western blot analysis.

DNA damage-inducing drug treatment. WT, RNF20-1and $\mathrm{RNF} 40^{-/-}$HCT116 cell lines were treated with DNA damage-inducing drugs doxorubicin (Dox; $0.5 \mu \mathrm{M}$ ) and etoposide (VP-16; $100 \mu \mathrm{M}$ ) for $0,2.5$ and $5 \mathrm{~h}$ prior to western blot analysis. WT, RNF20 ${ }^{-/}$, RNF40/-, p53/- HCT116 cell lines and p53 $^{-/}$HCT116 transfected with pCDNA3.1-HA-p53 R282W or pCDNA3.1-HA-p53 WT cells were treated with Dox $(0.25 \mu \mathrm{M})$ for $12 \mathrm{~h}$ prior to RT-qPCR detection.

Statistical analysis. Data are presented as the mean \pm SD. One-way ANOVA followed by Bonferroni's correction was used to determine the statistical differences among the experimental groups. GraphPad Prism version 6.02 for Windows (GraphPad Software, Inc.) and ImageJ 1.42q software with Java 1.6.0_12 (64-bit) (National Institutes of Health) were used for data and statistical analysis. $\mathrm{P}<0.05$ was considered to indicate a statistically significant difference.

\section{Results}

RNF20, RNF40 and WAC interacts with p53 through the coiled-coil domain. p53 has been demonstrated to be functionally associated with genomic DNA and involved in chromatin modifications (31). Furthermore, the chromatin RNF20/RNF40/WAC remodeler complex has been reported to mediate the protein expression levels of p53 $(32,33)$. Therefore, endogenous co-IP and western blot assays were performed in U2OS cell lysates to detect the interaction between p53 and the RNF20/RNF40/WAC complex. The results revealed that p53 interacted with RNF20, RNF40 and WAC (Fig. 1A). Next, a series of internal deletion mutations within RNF20, RNF40 and WAC were created, as indicated in the structure images to map the interaction regions in RNF20, RNF40 and WAC with p53 (Fig. 1B-D). As demonstrated in Fig. 1B-D, the D4 mutant of RNF20, the D7 and D8 mutants of RNF40 and the D7 mutant of WAC disrupted the interaction with p53, indicating that the coiled-coil motif of RNF20 and RNF40 in the corresponding areas recognized the p53 protein. However, p53, RNF20 and RNF40 all interacted with WAC through the WD7 mutant; therefore, it was hypothesized that either p53 interacted with WAC directly, or the RNF20/RNF40 dimer functioned as a bridge to connect p53 and WAC.

Mapping the specific p53 binding site with RNF20 and RNF40. Subsequently, the missense mutation in the DBD, which has been associated with $\sim 50 \%$ of all types of cancer, was investigated. To determine whether the mutation sites in the p53 DBD mediated the interaction with RNF20 and RNF40, a WT recombinant plasmid, pCDNA3.1-HA-p53, was used as a template, and two partial deletion mutants in DBD domain including $\triangle \mathrm{aa}$. 101-200 and $\Delta \mathrm{aa}$. 201-300 were constructed (Fig. 2A and B) and transfected into the U2OS cell line. The cell lysates were examined using co-IP and western blot assays. The results demonstrated that the $\Delta$ aa. 201-300 mutation prevented p53 from interacting with RNF20 and RNF40 (Fig. 2B), suggesting that the p53 C-terminal of DBD may be crucial for the interaction with RNF20 and RNF40. Next, a series of plasmids with high frequency cancer-related p53 missense mutations within the p53 C-terminal of DBD were constructed (Fig. 2C). A total of eight plasmids, including HA-p53WT, HA-p53R248W, HA-p53R248Q, HA-p53R249S, HA-p53R273C, HA-p53R273H, HA-p53R282W and p53R248QR273C, were transfected into the 293T cell line, and the interactions between p53 and RNF20 were examined using co-IP and western blot assays. The results demonstrated that the R282W mutation in the p53 DBD prevented p53 from interacting with RNF20 (Fig. 2B). Thus, the key site for the p53 interaction with RNF20 was R282.

RNF20/RNF40 complex maintains the activities of p53 and its target genes. To further investigate the role of RNF20 
A
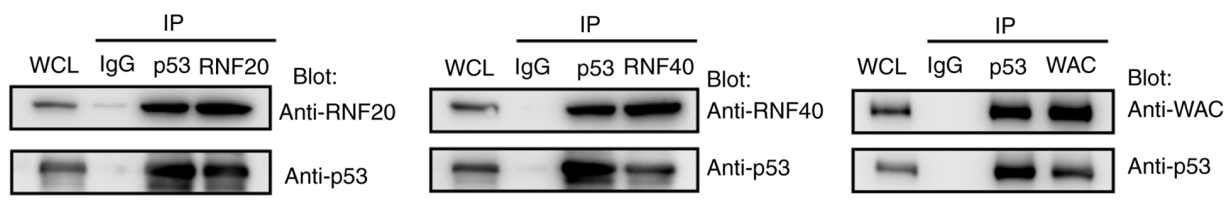

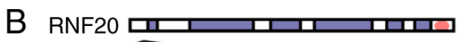
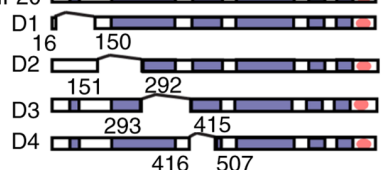

D5 משח

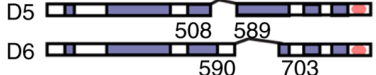

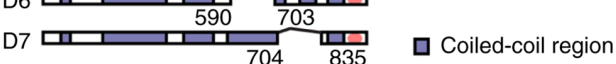

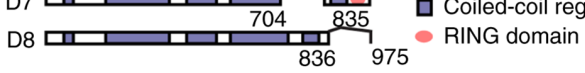

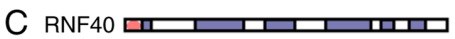
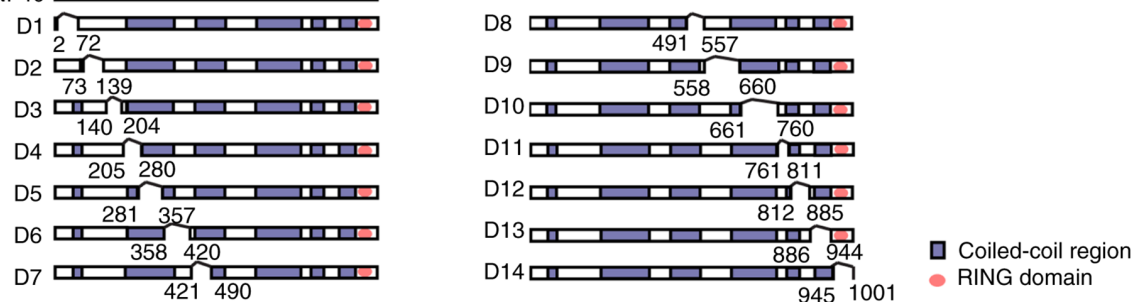

HA-RNF40
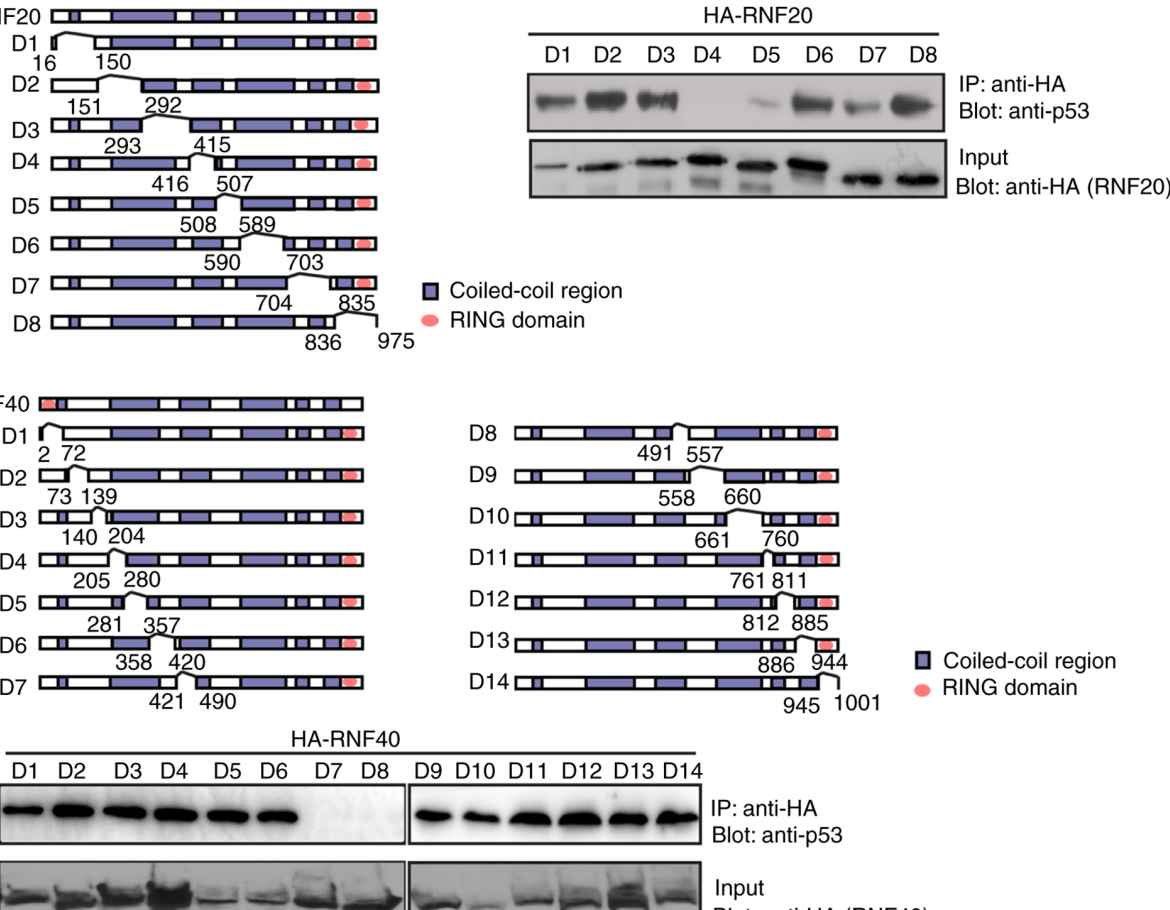

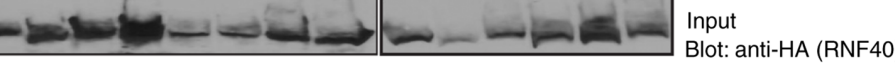

D WAC매

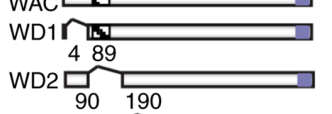

WD2 ${ }_{90}^{489}$

WD3 प्रण

WD5 270

WD6 प $498 \quad 558$ WW domain

WD7 प

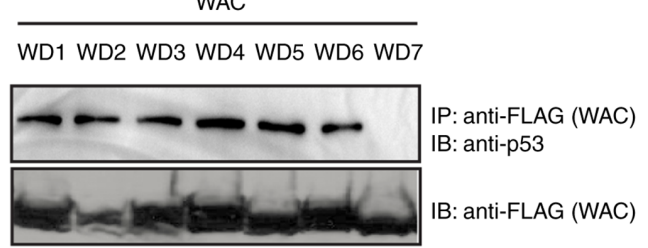

Figure 1. Mapping the interaction region of the RNF20/RNF40/WAC complex with p53. (A) Co-IP and western blot analysis of the endogenous interaction between p53 and RNF20/RNF40/WAC complexes. (B and C) Extracts from the U2OS cells transiently expressing HA-tagged wild-type (B) RNF20 or (C) RNF40 and their internal deletion mutations. Cell lysates were subjected to co-IP, followed by western blot analysis with anti-p53 and anti-HA antibodies. (D) FLAG-tagged wild-type WAC and its deletion mutations were transiently expressed in the U2OS cell line. The interaction between WAC and p53 was examined using Co-IP and western blot assays with anti-p53 and anti-FLAG antibodies. RNF, ring finger; WAC, WW domain-containing adaptor with coiled-coil; co-IP, co-immunoprecipitation; WCL, whole cell lysate; IB, immunoblot; D, deletion; WD, WAC deletion.

and RNF40 in p53 stabilization and transcription of p53 target genes, p53 protein expression levels were analyzed using western blot analysis, and the relative mRNA expression levels of p53, p21, PUMA, mash1 and Oct4 genes were determined using RT-qPCR in RNF20- or RNF40-knockout cells. CRISPR/Cas9 was used to knock out RNF20 and RNF40 in the HCT116 WT cell line, and the RNF20-- and RNF40 ${ }^{-/}$HCT116 cell lines were successfully constructed (Fig. 3A). The HCT116 WT, RNF20/- and RNF40 ${ }^{-/-}$HCT116 cell lines were treated with DNA damage-inducing drugs Dox $(0.5 \mu \mathrm{M})$ and VP-16 $(100 \mu \mathrm{M})$ for $0,2.5$ and $5 \mathrm{~h}$. Western blot analysis results demonstrated that following RNF20- or RNF40-knockout in the HCT116 cell lines, the protein expression levels of p53 were significantly reduced compared with those in the WT cells. Following DNA damage induced by Dox and VP-16, the p53 protein expression levels were increased in all the cell lines compared with those in the cells without Dox or VP-16 treatment; however, the $\mathrm{p} 53$ protein expression levels were lower in the RNF20/- and RNF40 ${ }^{-/-}$HCT116 cells lines compared with those in the WT cell line (Fig. 3B and C). Thus, RNF20 and RNF40 stabilized the p53 protein expression level. To determine the effects of the RNF20/RNF40 complex on the mRNA expression levels of p53 and its downstream genes, the HCT116 WT, RNF20 ${ }^{-/}$and RNF40 ${ }^{-/}$cell lines were treated with Dox $(0.25 \mu \mathrm{M} ; 12 \mathrm{~h})$, and the relative mRNA expression levels of the transcription targets of p53 were detected using RT-qPCR. The results demonstrated that following RNF20and RNF40-knockout, the mRNA expression levels of p53, 
A 553 protein structure and its missense mutations

\begin{tabular}{|l|l|l|l|l|l|l|}
\hline TAD & PRD & DBD & & & \\
\hline
\end{tabular}

B
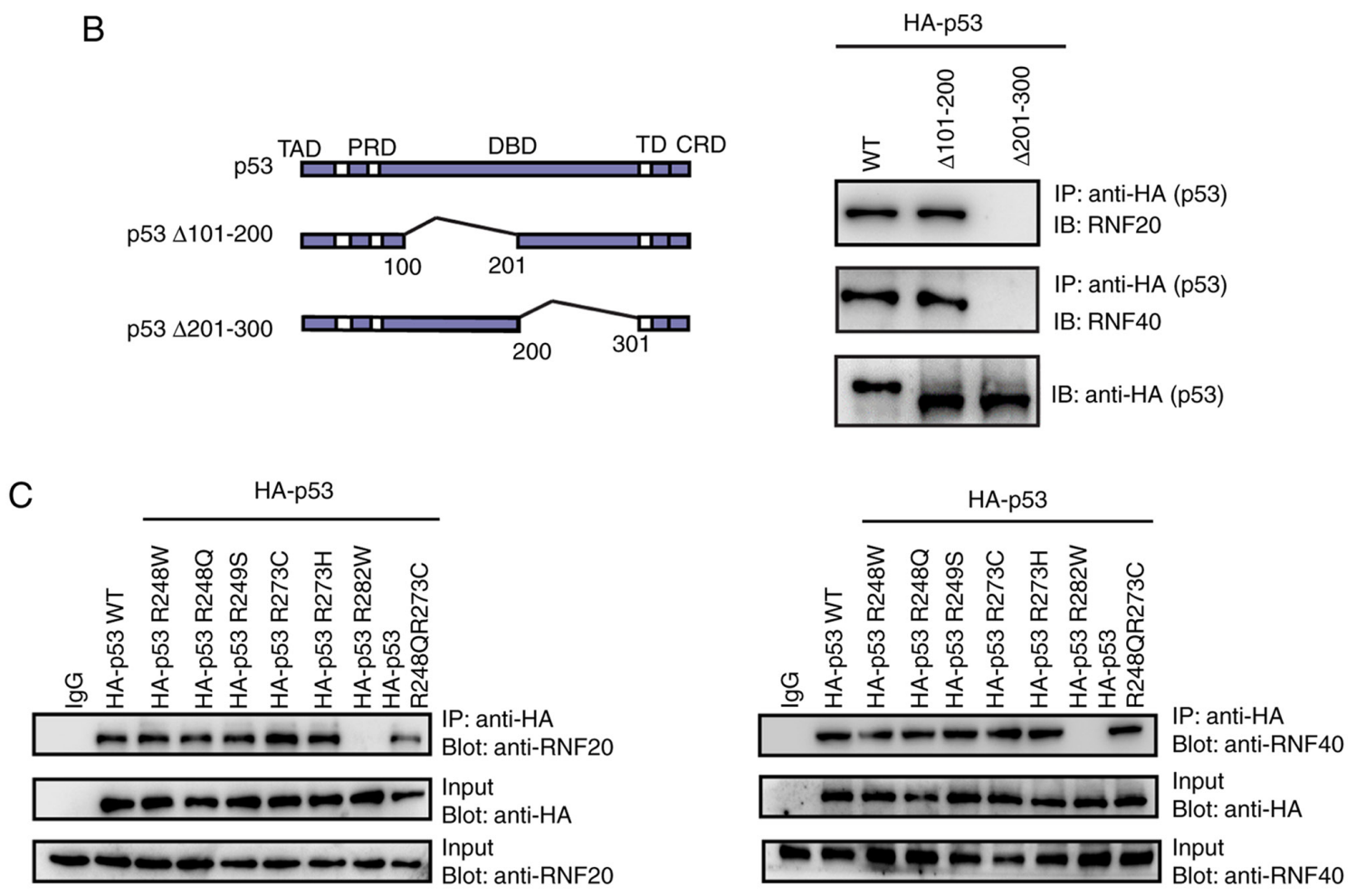

Figure 2. Mapping the key binding site in p53 with RNF20 and RNF40. (A) The location of the p53 missense mutations associated with cancer in the DNA-binding domain. (B and C) The 293T cell line was transfected with pCDNA3.1-HA-p53 plasmids with WT, partial internal deletion and the indicated missense mutations. Co-IP and western blot analyses were used to determine the exact site in p53, which interacted with RNF20 and RNF40. RNF, ring finger; co-IP, co-immunoprecipitation; IB, immunoblot; WT, wild-type. TAD, transcriptional activation domain; PRD, proline-rich domain; DBD, DNA-binding domain; TD, tetramerization domain; $\mathrm{CRD}$, carboxy-terminal regulatory domain.

p21, PUMA, mash1 and Oct4 in these cell lines without Dox treatment were significantly lower compared with those in the WT cells without Dox treatment. Following induction of DNA damage, the increases in the levels of p53 and its target genes in RNF20 ${ }^{-/-}$and RNF40 ${ }^{-/}$HCT116 cells lines treated with Dox were inhibited compared with those in WT HCT116 cells treated with Dox (Fig. 3D). Taken together, these results suggested that the RNF20/RNF40 complex stabilized the p53 protein expression levels and maintained the mRNA expression of its p53 target genes.

Mutation in 53 associated with RNF20 interaction suppresses the $m R N A$ expression levels of 53 target genes. The loss of the RNF20/RNF40 dimer downregulated the mRNA expression levels of the p53 target genes both in normal conditions and following DNA damage; however, it was unclear whether the p53 key binding site for RNF20, R282, mediated the inhibition of the p53 target gene expression following DNA damage. To investigate this, the pCDNA3.1-HA-p53 R282W plasmid was transfected into the p53/- HCT116 cell line (Fig. 4A). Following Dox $(0.5 \mu \mathrm{M})$ treatment for $12 \mathrm{~h}$, the mRNA expression levels of p21 in the WT cells were significantly increased compared with those in the untreated WT cells. The mRNA expression levels of $\mathrm{p} 21$ in the $\mathrm{p} 53^{-/-}$HCT116 cells were markedly reduced compared with those in the WT cells, and the p21 levels of p53 ${ }^{-/}$HCT116 cells treated with Dox were reduced compared with those in WT cells treated with Dox; however, the transfection of the pCDNA3.1-HA-p53 WT plasmid in p53 $3^{-/}$HCT116 cells rescued the mRNA expression level of $\mathrm{p} 21$ compared with $\mathrm{p} 53^{--}$HCT116 cells. In addition, the mRNA expression levels of p21 were lower in the pCDNA3.1-HA-p53 R282W-transfected p53-- HCT116 cells compared with those in cells transfected with pCDNA3.1-HA-p53 WT, which indicated that the p53 R282 site mediated the mRNA expression of p21 (Fig. 4B). The results of the PUMA, Oct4 and Mash1 mRNA expression level analysis were consistent with those of p21 (Fig. 4B). These results demonstrated that the R282W mutation resulted in the inability of p53, to bind to RNF20, which further inhibited the downstream target genes of p53.

\section{Discussion}

p53 activates a variety of transcriptional targets in response to cellular stress or DNA damage (4). It has been reported that the RNF20/RNF40/WAC complex regulates the transcription of the p53 target genes by regulating H2Bub1 under genotoxic 
A

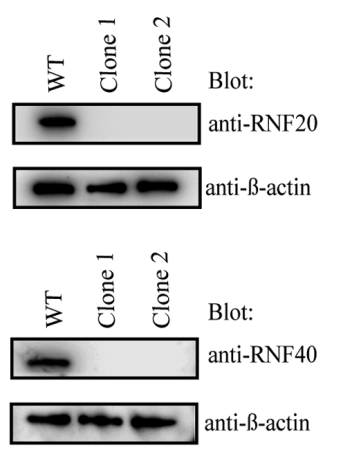

$\mathrm{B}$

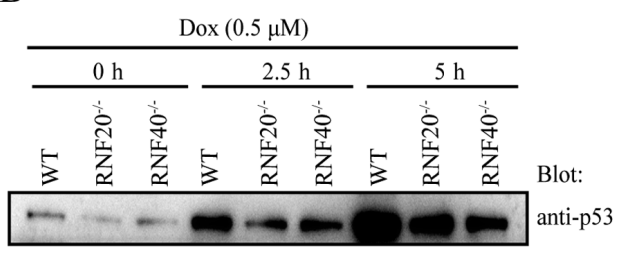

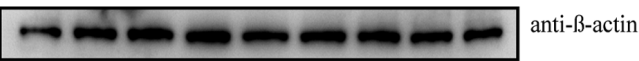

$\mathrm{C}$

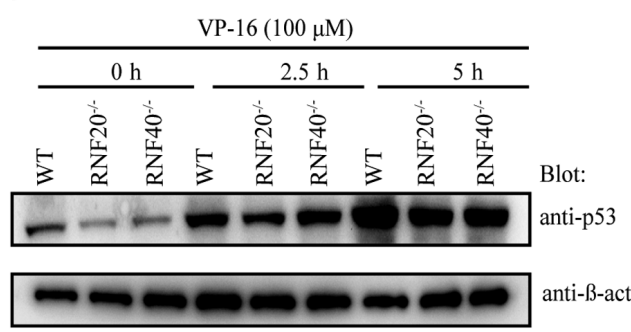

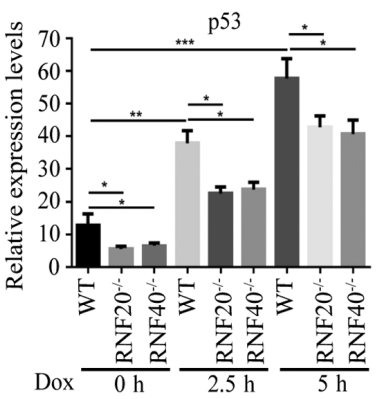

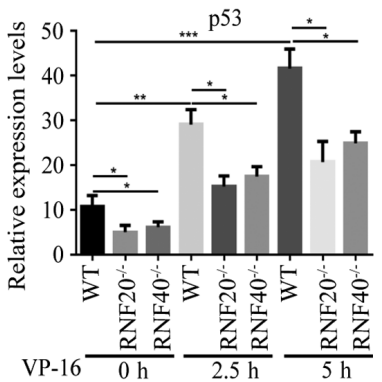

$\mathrm{D}$
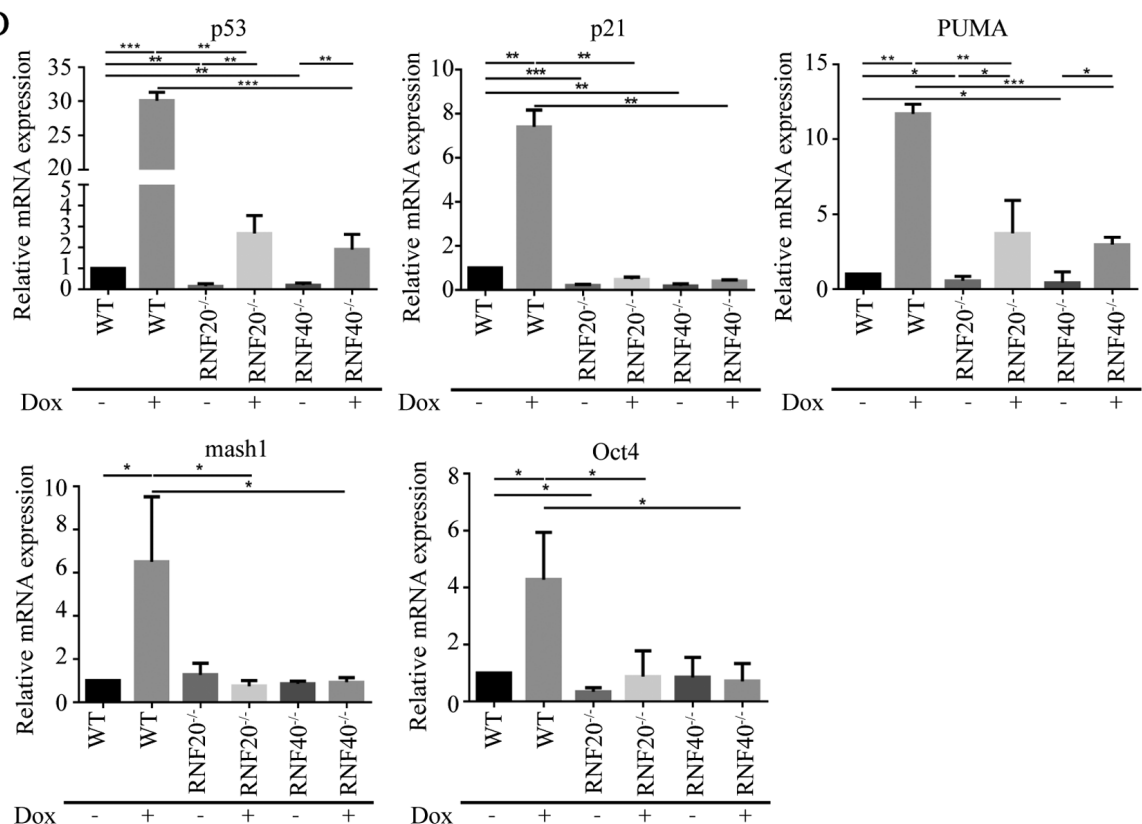

Figure 3. RNF20/RNF40 dimer maintains the activity of 553 and its target genes. (A) RNF20 ${ }^{-/}$and RNF40 $0^{-/} \mathrm{HCT} 116$ cell lines were successfully constructed using CRISPR/Cas9. Clones 1 and 2 were two samples from the RNF20 ${ }^{-/} \mathrm{HCT} 116$ cell line, and clones 3 and 4 were from the RNF40 ${ }^{-/} \mathrm{HCT} 116$ cell line. $(\mathrm{B}$ and $\mathrm{C})$ The protein expression levels of $\mathrm{p} 53$ were analyzed using western blot analysis in the HCT116 WT, RNF20 $0^{-/}$and RNF40 $0^{--}$cell lines following treatment with $0.5 \mu \mathrm{M}$ Dox and $100 \mu \mathrm{M}$ VP-16 for 0, 2.5 and $5 \mathrm{~h}$. (D) The relative mRNA expression levels of p53, p21 PUMA, mash1 and Oct4 in the WT, $\mathrm{RNF}_{2} 0^{-/}$and RNF40 $0^{-/} \mathrm{HCT} 116$ cell lines with or without doxorubicin treatment from three independent experiments were quantified using reverse transcription-quantitative PCR. The data are presented as the mean $\pm \mathrm{SD} . \mathrm{n}=3 .{ }^{*} \mathrm{P}<0.05,{ }^{* *} \mathrm{P}<0.01$ and ${ }^{* * *} \mathrm{P}<0.001$. RNF, ring finger; WT, wild-type; Dox, doxorubicin; VP-16, etoposide; PUMA, p53-upregulated modulator of apoptosis; mash1, Achaete-Scute homolog 1; Oct4, octamer-binding protein 4.

stress (14). H2Bub1 is conserved in the evolution process from yeast to mammals (34). Mammalian RNF20 and RNF40 have been demonstrated to be homologous to the E3 ubiquitin ligase Bre1 (BRE1) gene in yeast, forming a protein dimer, which synergistically interacts with E2 ubiquitinase RAD6 to catalyze histone $\mathrm{H} 2 \mathrm{~B}$ ubiquitination (35). WAC is a functional partner of RNF20/RNF40, which interacts with the coiled-coil region of RNF20/RNF40 to mediate H2Bub1 (21). The present study aimed to investigate the interaction domain between the RNF20/RNF40/WAC complex and p53, as well as the role of this complex gene transcription regulation.
The coiled-coil domains in RNF20, RNF40 and WAC have been reported to be highly conserved regions, as well as the WW domain of WAC, suggesting that these areas may serve important biological functions (33). Notably, the interaction between RNF20/40 and WAC is also mediated through the coiled-coil motif (21). The results of the present study demonstrated that p53 not only interacted with RNF20 and RNF40, consistent with a previous report by $\mathrm{Wu}$ et al (27), but also with WAC directly or through the RNF20/RNF40 dimer. p53 bound to the coiled-coil regions of RNF20, RNF40 and WAC. In addition, a series of deletion mutants of p53 were constructed 

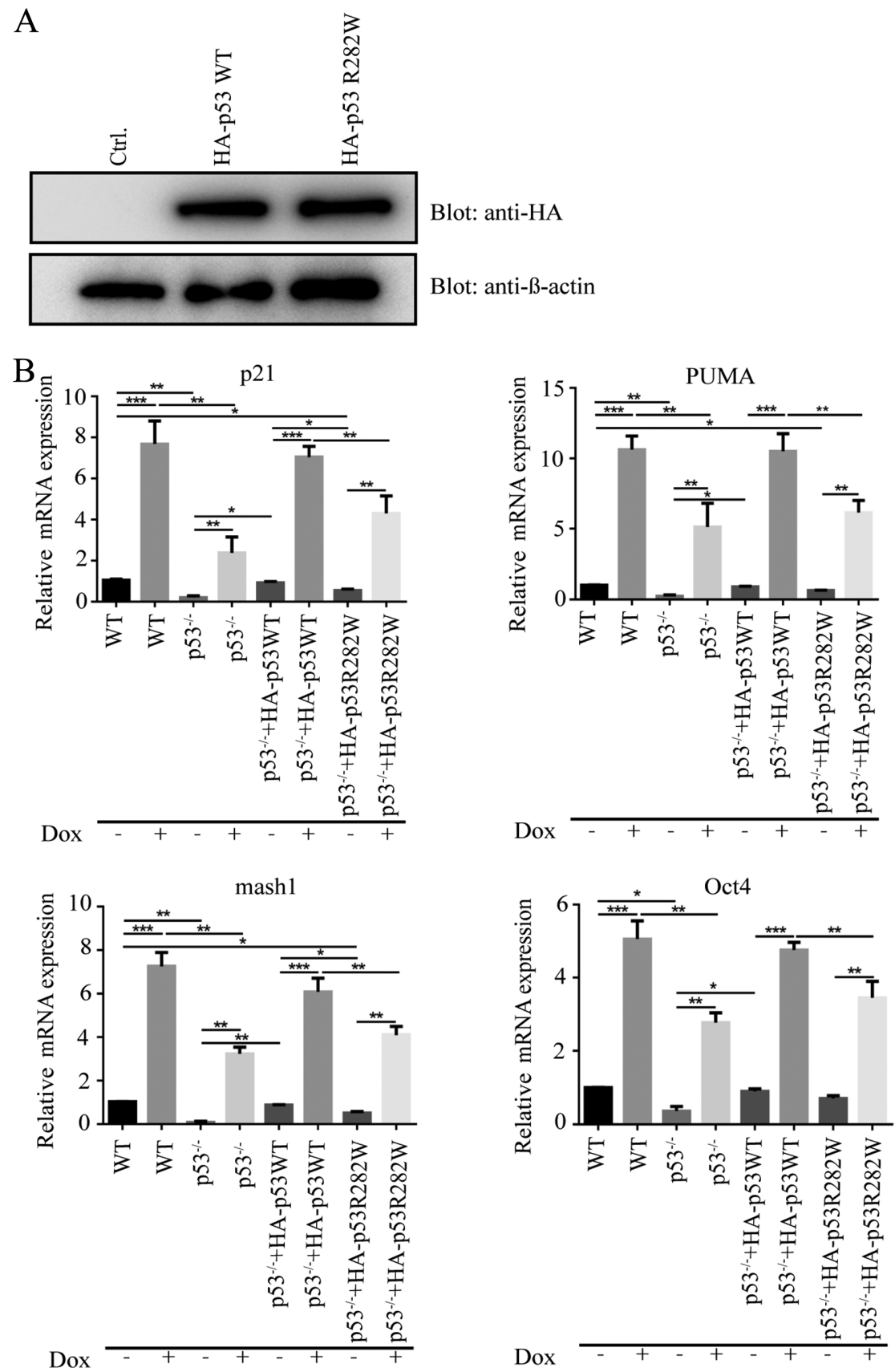

Figure 4. RNF20/RNF40 dimer regulates p53 target gene expression. (A) Western blot analysis of the transfection of HCT116 cell line was transfected with pCDNA3.1HA-p53 WT and pCDNA3.1HA-p53R282W plasmids. (B) Reverse transcription-quantitative PCR analysis of the effect of the p53 missense mutation on the regulation of its target genes in HCT116 WT, p53 ${ }^{-/}$HCT116 acquired using CRISPR/Cas 9 technology and p53 $3^{-/}$HCT116 cells transfected with pCDNA3.1HA-p53 WT or pCDNA3.1HA-p53R282W with or without Dox treatment. The data are presented as the mean \pm SD. $n=3$. " $\mathrm{P}<0.05$, ${ }^{* *} \mathrm{P}<0.01$ and ${ }^{* * * *} \mathrm{P}<0.001$. RNF, ring finger; WT, wild-type; Dox, doxorubicin; PUMA, p53-upregulated modulator of apoptosis; mash1, Achaete-Scute homolog 1; Oct4, octamer-binding protein 4.

in the present study, and the results demonstrated that other than the CTD region interacting with RNF20, the C-terminal part of the p53 DBD also interacted with RNF20/RNF40.

p53 initiates the transcription of genes associated with cell cycle arrest, cell senescence, apoptosis, metabolism, DNA repair and other processes under cell stress $(36,37)$. The loss of p53 function is primarily attributed to mutations, including gene fragment deletions, insertions, missense mutations and loss of heterozygosity (38). Missense mutations caused by single nucleotide polymorphisms account for $>80 \%$ of the total p53 mutations (39). Among these p53 missense muta- tions, $97 \%$ are point mutations and occur in the DBD (40). Mutations at the following positions occur at high frequency in cancer and are also termed hotspot mutations: R175H, R248Q, R248W, R249S, R273H, R273C and R282W (41). The present study focused on the specific sites interacting with the RNF20/RNF40 dimer close to the C-terminal region of the $\mathrm{p} 53 \mathrm{DBD}$. These mutations were screened by generating a series of missense mutants, and the results of the co-IP assay revealed that R282W was a key site for interaction with the RNF20/RNF40/WAC complex. Since human RNF20 and RNF40 share sequence homology with BRE1 in 
Saccharomyces cerevisiae, the key binding sites of p53 interacting with RNF20 and RNF40 are the same (42).

p53 activity is regulated by numerous post-translational modifications associated with protein chaperones, regulatory factors and chromatin remodelers (43). During the genotoxic stress response, p53 induces the transcription of a series of target genes, such as p21, growth arrest and DNA damage 45, Mdm2 proto-oncogene and PUMA (44). In the present study, RNF20 and RNF40 were demonstrated to be essential for maintaining p53 protein and mRNA expression levels, as well as those of its downstream genes, following DNA damage induction. Therefore, the RNF20/RNF40/WAC complex may be an important regulator of gene transcription associated with DNA damage response. Mutated p53 loses the function of cell cycle arrest, induction of apoptosis, mediation of cell senescence, repair of mismatched DNA bases and preservation of genomic stability (31). In addition, mutated p53 acquires a series of functions similar to those of oncogenes, such as transcription of target genes to accelerate cancer progression, enhancement of cancer cell chemical resistance and prevention of apoptosis; such mutations are termed gain-of-function (GOF) mutations (45). The results of the present study demonstrated that the R282 mutation, which was identified to be at the key binding site of p53 to RNF20 and RNF40, eliminated the induction of the p53 target genes. Thus, RNF20 cannot adequately maintain the stability of p53 and its downstream genes. However, the detailed mechanism remains unclear.

Previous studies have demonstrated that the R282W mutation is an important cause of p53 GOF, since it alters the protein-protein interaction ability and DNA-binding function of p53 (46-48). This p53 GOF mutant interacts with p63 and p73, which is involved in chemoresistance and anticancer drug metabolism through cytochrome P450 3A4 induction (49). The R282W mutation also contributes to the epithelial-mesenchymal transition by suppressing Kruppel-like factor 17 and promotes cancer cell invasion by microRNA-155 induction $(50,51)$. The R282 mutation has also been identified in patients with Li-Fraumeni syndrome and is enriched in bone tumors compared with bone tissues from healthy subjects (52).

Post-translational modification of proteins is a common mechanism in various cell signaling pathways in eukaryotic cells (43). For instance, poly(ADP) ribose polymerase 1-mediated poly-ribosylation is an essential component of base excision repair pathways, and ataxia telangiectasia and Rad3-related protein/ataxia telangiectasia mutated-mediated phosphorylation of protein kinase checkpoint kinase 1 (CHK1)/CHK2 are required in DNA double-strand break repair pathways $(43,53)$. Ubiquitination serves a crucial role in the cell cycle and proliferation, regulating the cellular levels of cytokines and coordinating oncogene transcription and the DNA damage response (22). According to a recent study, defects in ubiquitination are associated with human malignancies, such as ovarian, breast and colorectal cancer $(26,54,55)$. Hooda et al (55) have reported that RNF20 deficiency contributes to high-grade serous ovarian carcinoma initiation, and a study by Tarcic et al (26) revealed that RNF20 represses NF- $x$ B and its downstream inflammatory cytokines, which inhibits cancer cells proliferation and migration in basal-like breast tumors. Tarcic et al (54) have also reported that RNF20 $0^{+/-}$mice are predisposed to inflammation-associated colorectal cancer, and tissues from human colorectal tumors exhibit downregulation of RNF20/RNF40 and H2Bub1 in both the epithelium and the stroma. Therefore, mutations or low expression levels of the RNF20/RNF40/WAC complex, along with the dysregulation of deubiquitinases and cyclin-dependent kinases have been reported to affect tumorigenic pathways (55). Taken together, the R282W mutation and the RNF20/RNF40/WAC complex are associated with the clinical prognosis of patients with colorectal or breast cancer $(47,56)$. Previous studies have hypothesized that decitabine may maintain RNF20 expression to restore $\mathrm{H} 2 \mathrm{Bub} 1$ expression levels for treating primary breast carcinoma $(22,57)$. Furthermore, proteasome inhibitors may be considered for the treatment of early stage tumors $(58,59)$. In addition, the p53 R282 mutation has been predicted to be a potential biomarker for cancer prognosis, as it is associated with radioresistance, and patients with bladder cancer harboring the p53 R282 mutation have been shown to exhibit a shorter survival time compared with that in patients with nonsense mutations $(47,60,61)$. However, whether the p53 R282W mutant and the RNF20/RNF40/WAC complex collaboratively contribute to tumor development and progression requires further investigation.

\section{Acknowledgements}

The authors would like to thank Miss Jie Ren and Miss Yuyu Jiang (Shanghai Normal University, Shanghai, China) for revising the manuscript.

\section{Funding}

This study was supported by The National Natural Science Foundation of China (grant nos. 81572775 and 81773004 to FZ) and the Program for Professor of Special Appointment (Eastern Scholar) at Shanghai Institutions of Higher Learning (grant no. TP2014055 to FZ).

\section{Availability of data and materials}

The datasets used and/or analyzed during the current study are available from the corresponding authors on reasonable request.

\section{Authors' contributions}

$\mathrm{DM}, \mathrm{KG}$ and $\mathrm{DZ}$ performed the experiments and created the figures. DM wrote the manuscript. $\mathrm{CZ}$ contributed to the analysis and interpretation of the data and the revision of the manuscript. CS and FZ conceived the project, designed the experiments, and analyzed and interpreted the data. DM, KG and FZ confirm the authenticity of all the raw data. All authors read and approved the final manuscript.

\section{Ethics approval and consent to participate}

Not applicable.

\section{Patient consent for publication}

Not applicable. 


\section{Competing interests}

The authors declare that they have no competing interests.

\section{References}

1. Bieging KT, Mello SS and Attardi LD: Unravelling mechanisms of p53-mediated tumour suppression. Nat Rev Cancer 14 359-370, 2014

2. Joerger AC and Fersht AR: Structural biology of the tumor suppressor p53. Annu Rev Biochem 77: 557-582, 2008.

3. Joerger AC and Fersht AR: Structural biology of the tumor suppressor p53 and cancer-associated mutants. Adv Cancer Res 97: 1-23, 2007.

4. Ozaki T and Nakagawara A: Role of p53 in Cell Death and Human Cancers. Cancers (Basel) 3: 994-1013, 2011.

5. Joerger AC and Fersht AR: Structure-function-rescue: The diverse nature of common p53 cancer mutants. Oncogene 26 $2226-2242,2007$.

6. Riley T, Sontag E, Chen P and Levine A: Transcriptional control of human p53-regulated genes. Nat Rev Mol Cell Biol 9: 402-412, 2008

7. Allen MA, Andrysik Z, Dengler VL, Mellert HS, Guarnieri A, Freeman JA, Sullivan KD, Galbraith MD, Luo X, Kraus WL, et al: Global analysis of p53-regulated transcription identifies its direct targets and unexpected regulatory mechanisms. eLife 3: e02200, 2014.

8. Dai C and Gu W: p53 post-translational modification: Deregulated in tumorigenesis. Trends Mol Med 16: 528-536, 2010.

9. Bernard M, Yang B, Migneault F, Turgeon J, Dieudé M, Olivier MA, Cardin GB, El-Diwany M, Underwood K, Rodier F, et al: Autophagy drives fibroblast senescence through MTORC2 regulation. Autophagy 16: 2004-2016, 2020.

10. Ho CJ, Lin RW, Zhu WH, Wen TK, Hu CJ, Lee YL, Hung TI and Wang C: Transcription-independent and-dependent p53-mediated apoptosis in response to genotoxic and non-genotoxic stress. Cell Death Discov 5: 131, 2019.

11. Follis AV, Llambi F, Ou L, Baran K, Green DR and Kriwacki RW: The DNA-binding domain mediates both nuclear and cytosolic functions of p53. Nat Struct Mol Biol 21: 535-543, 2014.

12. Mrakovcic M and Frohlich LF: p53-mediated molecular control of autophagy in tumor cells. Biomolecules 8: 14, 2018.

13. Clapier CR and Cairns BR: The biology of chromatin remodeling complexes. Annu Rev Biochem 78: 273-304, 2009.

14. Cole AJ, Clifton-Bligh R and Marsh DJ: Histone H2B monoubiquitination: Roles to play in human malignancy. Endocr Relat Cancer 22: T19-T33, 2015.

15. Chandrasekharan MB, Huang F and Sun ZW: Histone H2B ubiquitination and beyond: Regulation of nucleosome stability, chromatin dynamics and the trans-histone H3 methylation. Epigenetics 5: 460-468, 2010.

16. Hammond-Martel I, Yu H and Affar B: Roles of ubiquitin signaling in transcription regulation. Cell Signal 24: 410-421, 2012.

17. Buetow L and Huang DT: Structural insights into the catalysis and regulation of E3 ubiquitin ligases. Nat Rev Mol Cell Biol 17: 626-642, 2016

18. Shiloh Y, Shema E, Moyal L and Oren M: RNF20-RNF40: A ubiquitin-driven link between gene expression and the DNA damage response. FEBS Lett 585: 2795-2802, 2011.

19. Zhang K, Wang J, Tong TR, Wu X, Nelson R, Yuan YC, Reno T, Liu Z, Yun X, Kim JY, et al: Loss of H2B monoubiquitination is associated with poor-differentiation and enhanced malignancy of lung adenocarcinoma. Int J Cancer 141: 766-777, 2017.

20. Shema E, Tirosh I, Aylon Y, Huang J, Ye C, Moskovits N, Raver-Shapira N, Minsky N, Pirngruber J, Tarcic G, et al: The histone H2B-specific ubiquitin ligase RNF20/hBRE1 acts as a putative tumor suppressor through selective regulation of gene expression. Genes Dev 22: 2664-2676, 2008

21. Zhang F and Yu X: WAC, a functional partner of RNF20/40, regulates histone $\mathrm{H} 2 \mathrm{~B}$ ubiquitination and gene transcription. Mol Cell 41: 384-397, 2011.

22. Johnsen SA: The enigmatic role of H2Bub1 in cancer. FEBS Lett 586: 1592-1601, 2012.

23. Sethi G, Shanmugam MK, Arfuso F and Kumar AP: Role of RNF20 in cancer development and progression - a comprehensive review. Biosci Rep 38: BSR20171287, 2018.
24. Chernikova SB, Razorenova OV, Higgins JP, Sishc BJ, Nicolau M, Dorth JA, Chernikova DA, Kwok S, Brooks JD, Bailey SM, et al: Deficiency in mammalian histone $\mathrm{H} 2 \mathrm{~B}$ ubiquitin ligase Brel (Rnf20/Rnf40) leads to replication stress and chromosomal instability. Cancer Res 72: 2111-2119, 2012.

25. Lee JH, Jeon YG, Lee KH, Lee HW, Park J, Jang H, Kang M, Lee HS, Cho HJ, Nam DH, et al: RNF20 Suppresses Tumorigenesis by Inhibiting the SREBP1c-PTTG1 Axis in Kidney Cancer. Mol Cell Biol 37: e00265-17, 2017.

26. Tarcic O, Granit RZ, Pateras IS, Masury H, Maly B, Zwang Y, Yarden Y, Gorgoulis VG, Pikarsky E, Ben-Porath I, et al: RNF20 and histone H2B ubiquitylation exert opposing effects in Basal-Like versus luminal breast cancer. Cell Death Differ 24 694-704, 2017.

27. Wu C, Cui Y, Liu X, Zhang F, Lu LY and Yu X: The RNF20/40 complex regulates p53-dependent gene transcription and mRNA splicing. J Mol Cell Biol 12: 113-124, 2020.

28. Tzin V, Rogachev I, Meir S, Moyal Ben Zvi M, Masci T, Vainstein A, Aharoni A and Galili G: Tomato fruits expressing a bacterial feedback-insensitive 3-deoxy-D-arabino-heptulosonate 7-phosphate synthase of the shikimate pathway possess enhanced levels of multiple specialized metabolites and upgraded aroma. J Exp Bot 64: 4441-4452, 2013.

29. Kitzman JO, Starita LM, Lo RS, Fields S and Shendure J: Massively parallel single-amino-acid mutagenesis. Nat Methods 12: 203-206, $4 \mathrm{p}$ following 206, 2015.

30. Livak KJ and Schmittgen TD: Analysis of relative gene expression data using real-time quantitative PCR and the 2(-Delta Delta C(T)) method. Methods 25: 402-408, 2001

31. Stiewe T and Haran TE: How mutations shape p53 interactions with the genome to promote tumorigenesis and drug resistance. Drug Resist Updat 38: 27-43, 2018.

32. Shema E, Tirosh I, Aylon Y, Huang J, Ye C, Moskovits N, Raver-Shapira N, Minsky N, Pirngruber J, Tarcic G, et al: Corrigendum: The histone $\mathrm{H} 2 \mathrm{~B}$-specific ubiquitin ligase RNF20/hBRE1 acts as a putative tumor suppressor through selective regulation of gene expression. Genes Dev 31: 1926, 2017.

33. Hwang WW, Venkatasubrahmanyam S, Ianculescu AG, Tong A, Boone C and Madhani HD: A conserved RING finger protein required for histone $\mathrm{H} 2 \mathrm{~B}$ monoubiquitination and cell size control. Mol Cell 11: 261-266, 2003.

34. Nakamura K, Kato A, Kobayashi J, Yanagihara H, Sakamoto S, Oliveira DV, Shimada M, Tauchi H, Suzuki H, Tashiro S, et al: Regulation of homologous recombination by RNF20-dependent H2B ubiquitination. Mol Cell 41: 515-528, 2011.

35. Kao CF, Hillyer C, Tsukuda T, Henry K, Berger S and Osley MA: Rad6 plays a role in transcriptional activation through ubiquitylation of histone H2B. Genes Dev 18: 184-195, 2004.

36. Tang Q, Su Z, Gu W and Rustgi AK: Mutant p53 on the path to metastasis. Trends Cancer 6: 62-73, 2020.

37. Vousden KH and Prives C: Blinded by the Light: The growing complexity of p53. Cell 137: 413-431, 2009.

38. Li H, Zhang J, Tong JHM, Chan AWH, Yu J, Kang W and To KF Targeting the oncogenic p53 mutants in colorectal cancer and other solid tumors. Int J Mol Sci 20: 5999, 2019.

39. Hanel W, Marchenko N, Xu S, Yu SX, Weng W and Moll U: Two hot spot mutant p53 mouse models display differential gain of function in tumorigenesis. Cell Death Differ 20: 898-909, 2013.

40. Cho Y, Gorina S, Jeffrey PD and Pavletich NP: Crystal structure of a p53 tumor suppressor-DNA complex: Understanding tumorigenic mutations. Science 265: 346-355, 1994.

41. Petitjean A, Mathe E, Kato S, Ishioka C, Tavtigian SV, Hainaut P and Olivier M: Impact of mutant p53 functional properties on TP53 mutation patterns and tumor phenotype: Lessons from recent developments in the IARC TP53 database. Hum Mutat 28 622-629, 2007.

42. Kumar P and Wolberger C: Structure of the yeast Bre1 RING domain. Proteins 83: 1185-1190, 2015.

43. Shanmugam MK, Arfuso F, Arumugam S, Chinnathambi A, Jinsong B, Warrier S, Wang LZ, Kumar AP, Ahn KS, Sethi G, et al: Role of novel histone modifications in cancer. Oncotarget 9: 11414-11426, 2017.

44. Menendez D, Nguyen TA, Freudenberg JM, Mathew VJ, Anderson CW, Jothi R and Resnick MA: Diverse stresses dramatically alter genome-wide $\mathrm{p} 53$ binding and transactivation landscape in human cancer cells. Nucleic Acids Res 41: 7286-7301, 2013.

45. Stein Y, Rotter V and Aloni-Grinstein R: Gain-of-function mutant p53: All the roads lead to tumorigenesis. Int J Mol Sci 20: $6197,2019$. 
46. Zhang Y, Coillie SV, Fang JY and Xu J: Gain of function of mutant p53: R282W on the peak? Oncogenesis 5: e196, 2016.

47. Xu J, Wang J, Hu Y, Qian J, Xu B, Chen H, Zou W and Fang JY: Unequal prognostic potentials of p53 gain-of-function mutations in human cancers associate with drug-metabolizing activity. Cel Death Dis 5: e1108, 2014.

48. Calhoun S and Daggett V: Structural effects of the L145Q $\mathrm{V} 157 \mathrm{~F}$, and R282W cancer-associated mutations in the p53 DNA-binding core domain. Biochemistry 50: 5345-5353, 2011.

49. Deyoung MP and Ellisen LW: p63 and p73 in human cancer: Defining the network. Oncogene 26: 5169-5183, 2007.

50. Ali A, Shah AS and Ahmad A: Gain-of-function of mutant p53: Mutant p53 enhances cancer progression by inhibiting KLF17 expression in invasive breast carcinoma cells. Cancer Lett 354: 87-96, 2014.

51. Neilsen PM, Noll JE, Mattiske S, Bracken CP, Gregory PA, Schulz RB, Lim SP, Kumar R, Suetani RJ, Goodall GJ, et al: Mutant p53 drives invasion in breast tumors through up-regulation of miR-155. Oncogene 32: 2992-3000, 2013.

52. Xu J, Qian J, Hu Y, Wang J, Zhou X, Chen H and Fang JY: Heterogeneity of Li-Fraumeni syndrome links to unequal gain-of-function effects of p53 mutations. Sci Rep 4: 4223, 2014.

53. Zhang Y and Hunter T: Roles of Chk1 in cell biology and cancer therapy. Int J Cancer 134: 1013-1023, 2014.

54. Tarcic O,Pateras IS, Cooks T, ShemaE,Kanterman J,Ashkenazi H, Boocholez H, Hubert A, Rotkopf R, Baniyash M, et al: RNF20 Links histone $\mathrm{H} 2 \mathrm{~B}$ ubiquitylation with inflammation and inflammation-associated cancer. Cell Rep 14: 1462-1476, 2016.

55. Hooda J, Novak M, Salomon MP, Matsuba C, Ramos RI, MacDuffie E, Song M, Hirsch MS, Lester J, Parkash V, et al: Early loss of histone H2B monoubiquitylation alters chromatin accessibility and activates key immune pathways that facilitate progression of ovarian cancer. Cancer Res 79: 760-772, 2019.
56. Zhang Y, Yao L, Zhang X, Ji H, Wang L, Sun S and Pang D: Elevated expression of USP22 in correlation with poor prognosis in patients with invasive breast cancer. J Cancer Res Clin Oncol 137: 1245-1253, 2011.

57. Minsky N, Shema E, Field Y, Schuster M, Segal E and Oren M: Monoubiquitinated $\mathrm{H} 2 \mathrm{~B}$ is associated with the transcribed region of highly expressed genes in human cells. Nat Cell Biol 10: 483-488, 2008

58. Prenzel T, Begus-Nahrmann Y, Kramer F, Hennion M, Hsu C, Gorsler T, Hintermair C, Eick D, Kremmer E, Simons M, et al: Estrogen-dependent gene transcription in human breast cancer cells relies upon proteasome-dependent monoubiquitination of histone H2B. Cancer Res 71: 5739-5753, 2011.

59. Mimnaugh EG, Chen HY, Davie JR, Celis JE and Neckers L: Rapid deubiquitination of nucleosomal histones in human tumor cells caused by proteasome inhibitors and stress response inducers: Effects on replication, transcription, translation, and the cellular stress response. Biochemistry 36: 14418-14429, 1997.

60. Skinner HD, Sandulache VC, Ow TJ, Meyn RE, Yordy JS, Beadle BM, Fitzgerald AL, Giri U, Ang KK and Myers JN: TP53 disruptive mutations lead to head and neck cancer treatment failure through inhibition of radiation-induced senescence. Clin Cancer Res 18: 290-300, 2012.

61. Okaichi K, Ide-Kanematsu M, Izumi N, Morita N, Okumura Y and Ihara M: Variations in sensitivity to ionizing radiation in relation to p53 mutation point. Anticancer Res 28 (5A): 2687-2690, 2008.

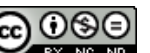

This work is licensed under a Creative Commons Attribution-NonCommercial-NoDerivatives 4.0 International (CC BY-NC-ND 4.0) License. 